\title{
Direct Energy Exchange Enhancement in Distributed Injection Light Gas Launchers
}

T. W. Alger, R. G. Finucane, J. P. Hall, B. M. Penetrante, T. M. Uphaus

April 6, 2000

U.S. Department of Energy

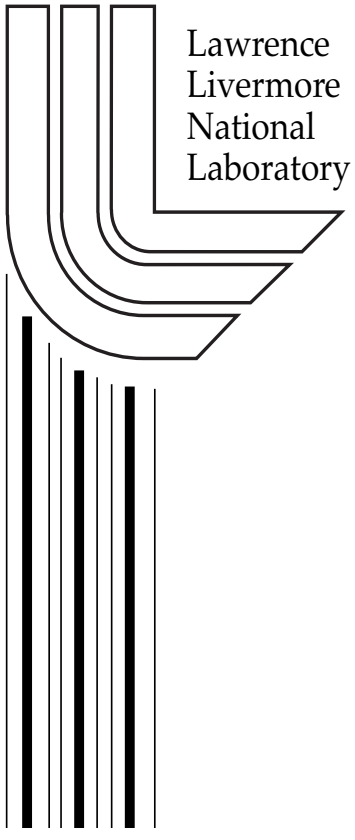




\section{DISCLAIMER}

This document was prepared as an account of work sponsored by an agency of the United States Government. Neither the United States Government nor the University of California nor any of their employees, makes any warranty, express or implied, or assumes any legal liability or responsibility for the accuracy, completeness, or usefulness of any information, apparatus, product, or process disclosed, or represents that its use would not infringe privately owned rights. Reference herein to any specific commercial product, process, or service by trade name, trademark, manufacturer, or otherwise, does not necessarily constitute or imply its endorsement, recommendation, or favoring by the United States Government or the University of California. The views and opinions of authors expressed herein do not necessarily state or reflect those of the United States Government or the University of California, and shall not be used for advertising or product endorsement purposes.

This work was performed under the auspices of the U. S. Department of Energy by the University of California, Lawrence Livermore National Laboratory under Contract No. W-7405-Eng-48.

This report has been reproduced directly from the best available copy.

Available electronically at http://www.doc.gov/bridge

Available for a processing fee to U.S. Department of Energy

And its contractors in paper from

U.S. Department of Energy

Office of Scientific and Technical Information

P.O. Box 62

Oak Ridge, TN 37831-0062

Telephone: (865) 576-8401

Facsimile: (865) 576-5728

E-mail: reports@adonis.osti.gov

Available for the sale to the public from

U.S. Department of Commerce

National Technical Information Service

5285 Port Royal Road

Springfield, VA 22161

Telephone: (800) 553-6847

Facsimile: (703) 605-6900

E-mail: orders@ntis.fedworld.gov

Online ordering: http://www.ntis.gov/ordering.htm

OR

Lawrence Livermore National Laboratory

Technical Information Department's Digital Library

http:/ / www.llnl.gov/tid/Library.html 
Development of High-Velocity Launcher Technology

Direct energy exchange enhancement in distributed injection light gas launchers. T. W. Alger, R. G. Finucane, J. P. Hall, B. M. Penetrante, T. M. Uphaus

\section{8-ERD-055}

It is not widely acknowledged or appreciated that conventional, two-stage light-gas launchers do not efficiently apply their high breech pressures to the design intent: accelerating the projectile. Our objective in this project was to carry out the analysis, design, construction, and testing of a new class of launchers that will address this limitation. Our particular application is to expand the pressure range of the conventional, two-stage gas launcher to overlap and validate the pressure regimes previously attainable only with shock waves generated by nuclear explosions, lasers, or multistage conventional explosions. That is, these launchers would have the capability to conduct - in a laboratory setting — high-velocity-impact, equation-of-state (EOS) measurements at up to 2-TPa (20 Mbar) pressure levels in high- $Z$ materials.

Our design entailed a new class of distributed-injection, gas-dynamic launchers that are designed to use a boat-tail projectile to overcome the fundamental gas-expansion phenomena known as escape velocity (the Riemann limit).

Our program included analytical, numerical, and experimental studies of the fast gas release flow technique that is central to the success of our approach. The analyses led us to believe that, in a typical configuration, the pressure will be effectively applied to the projectile in a time short relative to its few-microsecond traverse time; the experimental program we conducted during FY1999 supported these estimates. In addition, our program revealed dramatic increased efficiency in this process that was previously unknown to the launcher community.

The most fundamental practical restrictions on the performance of any gas launcher are the ability of the launcher to (1) contain pressure in a reservoir, and (2) effectively apply that pressure to the base of a moving projectile. Our gas-release test-fixture experiments showed that our design was capable of applying nearly twice the pressure to the projectile that is initially contained in the reservoir. This results deserves emphasis: whereas conventional guns apply a few percent of the reservoir pressure to a fast moving projectile, our design is paradoxically capable of applying nearly double the contained pressure. We later confirmed this experimental result analytically and related it to a type of direct energy exchange between unsteady fluid flows. This physical approach was the basis for the German V-1 "buzz bomb" of World War II; it has been applied to a limited number of commercial applications. (This work should not be confused with the German WW II distributed injection missile launchers.) Direct fluid-energy exchange has not previously been applied to any gas-launcher technology.

As a result of these discoveries, we estimate that a practical, $15 \mathrm{~km} / \mathrm{s}$, high-velocity launcher could be built using our direct-energy-exchange, distributed-injection approach. However, the radical nature of the results, the lack of confirming or allied work being carried out anywhere else, and the fact that it would take extensive time and resources to demonstrate targeted performance precluded further development. We plan to submit the results to a refereed journal to ensure that the work will not be lost to the launcher community. 


\section{Introduction}

The conventional two-stage light-gas gun has been used for many years for high-pressure equation of state (EOS) measurements by launching carefully designed projectiles at instrumented targets. The resulting impact generates intense shock waves, producing high dynamic pressures and temperatures in the targets, allowing equation of state measurements to be carried out at extreme states of matter. A conventional two-stage gun is limited to a velocity near $8 \mathrm{~km} / \mathrm{s}$ on a routine basis with planar projectiles (1). This velocity capability limits the pressures attainable in dynamic EOS experiments to about $600 \mathrm{GPa}$ (6 Mbar) in high-Z metals, whereas pressures to the $2 \mathrm{TPa}$ range are necessary to support the needs of EOS and other user communities.

Despite many attempts over the last forty years to enhance the performance of the two stage light gas gun, little sustained progress has been achieved. In the last few years, new theoretical understanding of the internal gas dynamics of guns has opened the possibility of overcoming the longstanding $8 \mathrm{~km} / \mathrm{s}$ limit.

An unsteady gas expansion process is the driving mechanism for the two-stage light gas gun. Compressed air, gunpowder, or other energy source accelerates a relatively heavy first stage piston, which compresses and heats the drive gas to a level determined by a burst diaphragm just ahead of the second stage projectile. The attainable velocity is based on the maximum pressure (and temperature) capability of the first stage barrel, or pump tube as it is called. When the diaphragm bursts, the pressure begins to accelerate the projectile down the barrel, leading to exponential pressure decay at the base of the projectile in the process. As the projectile continues to accelerate past the initial sound speed of the propellant, the projectile acceleration drops with the pressure, and the observed velocity plateau is reached.

In principle, one is free to increase gun performance by changing gas composition, initial fill temperature and pressure, piston and projectile masses and diameters, piston velocity profile, and so on. In practice, performance is limited by the maximum temperature and pressure capability of the pump tube and other gun components. As material limits are approached, high temperature, high-pressure gas erosion of the barrel lead to additional performance losses in practical applications (2).

The fundamental ballistic limits of conventional and light gas guns, and the inefficiency with which the high gas pressure is able to be applied to accelerating the projectile, has been known for hundreds of years. During the last century, the idea of distributing the high pressure source along the length of the barrel and applying it to the moving projectile as it passes by has been the subject of repeated theoretical and experimental attention, with little success being reported. Gilreath et. al. (3) pointed out the critical theoretical errors that motivated many of the previous failed attempts at distributed injection. This paper first suggested that the theoretical problems could be overcome by applying the pressure onto the side of the tapered or conical base of a projectile, rather than against the flat base. The history of these ideas and a first order theoretical exposition of the performance parameters of launchers are brilliantly described in a paper by Higgins (4).

Our program included analytical, numerical, and experimental studies of the fast gas release flow technique that is central to the success of the distributed injection approach. The analyses led us to believe that, in a typical configuration, the pressure will be effectively applied to the projectile 
in a time short relative to its few-microsecond traverse time. The experimental program we conducted supported these estimates. In addition, our program revealed some dramatic increased efficiencies in this process that were completely unexpected and previously unknown to the launcher community.

The present paper describes a project to experimentally and analytically study some of the crucial technical advances required for the distributed injection concept. We designed and tested two fast diaphragm-opening techniques, deciding on the basis of these tests to use an exploding bridge wire (EBW) driven by a high-voltage capacitor discharge. We designed and built a gas-release test fixture to study the rate of rise of pressure at a fixed distance from the EBW-fired bursting diaphragm. In parallel with this work, we designed and built a first-stage helium launcher, consisting of a pressure intensifier used to boost the helium-bottle pressure from 10 to $100 \mathrm{MPa}$, a 100-MPa gas breech, and a new, 5-m-long, 20-mm-diam barrel. We also designed a distributed-injection acceleration section and assembled a target chamber and associated velocity-measurement equipment (a velocity interferometry system for any reflector, or VISAR) into a complete launcher system.

\section{Conceptual design description}

This concept embodies a new approach toward overcoming the fundamental performance limitations of conventional powder guns and two stage light gas guns.

The conventional gas gun works by an unsteady expansion process. As the gas expands, and the projectile begins to accelerate, the drive pressure drops to only a few percent of the original value as the projectile reaches about twice the initial sound speed in the drive gas. One method to overcome this loss that has been proposed and tried over the years is to inject additional high pressure gas from a series of distributed reservoirs onto the base of the projectile as it traverses the length of the barrel.

The poor results experienced during past trials of distributed injection have been previously explained by difficulties involving critical timing, electrical interference, and other practical issues. Although these issues undoubtedly played a role in past failures, more fundamental matters went unrecognized. As a result of recent work done here at LLNL, at JPL, at McGill University, and elsewhere, it has become apparent that previous gas injection approaches have suffered from an inadequate theoretical understanding of the gas expansion loss mechanism. Previously, injection schemes were based on the implicit premise that the projectile was outdistancing the gas drive mechanism, whereas in reality, the projectile outruns the gas molecular flow. This subtle distinction explains past failures to attain projectile acceleration at high projectile velocities, and also points the way toward an alternative technical approach towards a distributed injection launcher.

This new concept involves injection of high-pressure gas onto a tapered "boattail" rear surface of the projectile. Because the apparent relative velocity of the tapered surface is reduced as the tangent of the boattail angle, this technique, if successful, has the capability of maintaining the drive pressure at substantially higher absolute projectile velocities. Theoretically, it should be possible to attain velocities 2-4 times that of existing guns at comparable pressure and temperature limits. As shown in the figure, in a properly designed distributed injection launcher will apply the maximum drive pressure at the optimum location: the projectile base. 

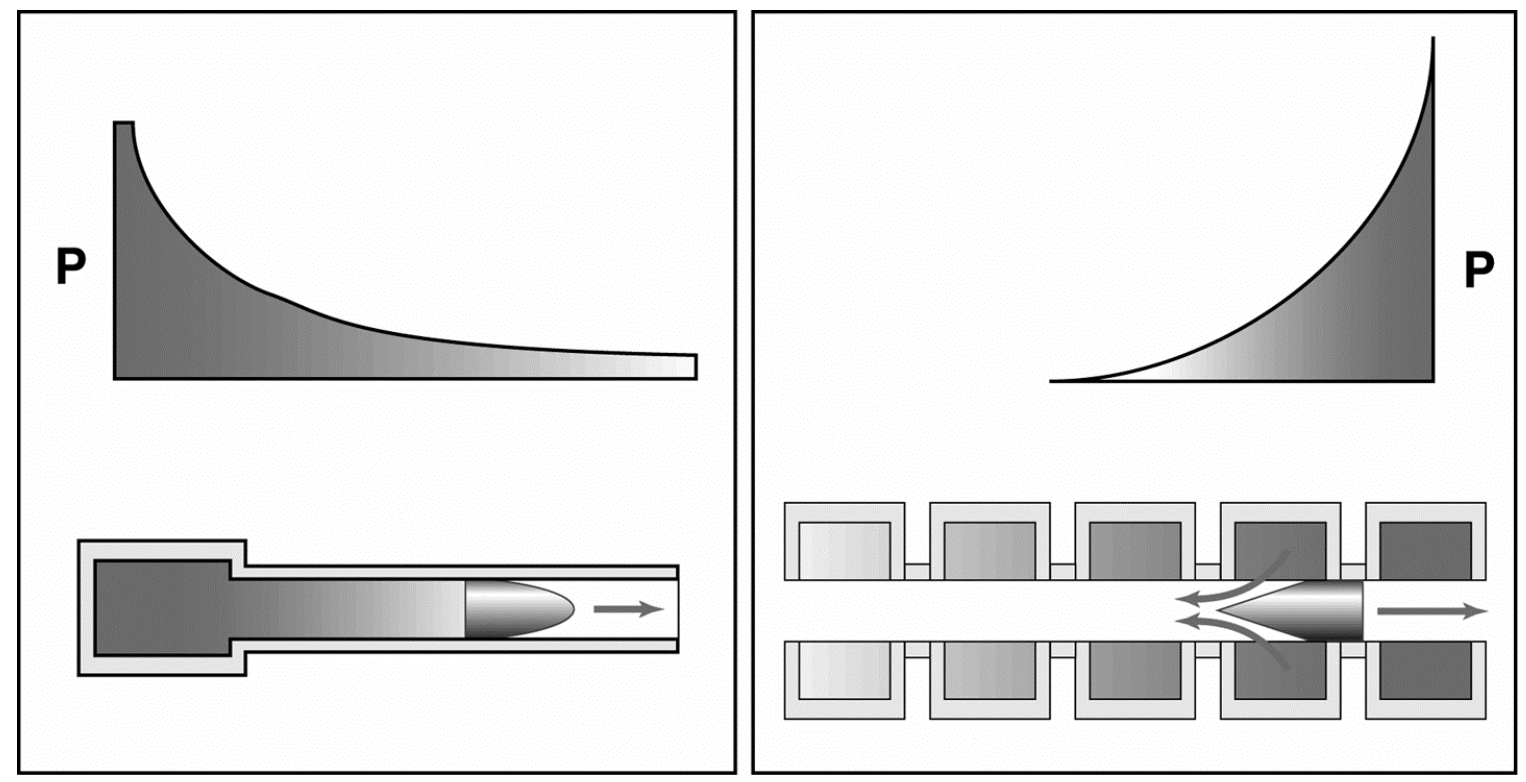

Figure 1. In conventional ballistics, the maximum pressure occurs where it does no good: at the breech. The projectile feels the lowest pressure in the system. A properly designed distributed injection launcher will apply the maximum pressure at the projectile.

In addition to a better theoretical understanding of the internal gas dynamics, we are also able to take advantage of substantial increases in the speed and performance of instrumentation and control systems. The critical engineering performance limit of the distributed injection launcher revolves around the ability to measure the position and velocity of the moving projectile on a sub microsecond time scale and release the high pressure gas supply onto the projectile expansion surface with similar temporal precision. Our approach takes advantage of fast electronics, optical detectors, and other advanced electro-mechanical systems that have evolved in the last 40 years.

\section{Mechanical Design Description}

We designed, fabricated, and assembled major components of the launcher, including a highpressure helium breech, first stage launch barrel, catch tank, and associated gas supply, instrumentation and control racks and equipment. Some of the equipment was salvaged from other experiments, such as the catch tank, mounting system, and instrument racks. We built components of the distributed injection section, but never assembled these latter components into a functioning system. Salient features of the launcher are summarized in Table 1. 
Table 1. Launcher design features

\begin{tabular}{|l|l|}
\hline First stage gas & Helium \\
\hline Breech pressure & $100 \mathrm{MPa}$ \\
\hline Breech dimensions & \\
\hline Breech volume & \\
\hline Barrel dimensions & $20 \mathrm{~mm}$ ID by $5 \mathrm{~m}$ long \\
\hline Projectile mass & \\
\hline Design maximum velocity, first stage & $2 \mathrm{~km} / \mathrm{s} ?$ \\
\hline Distributed Injection(DI) section length & \\
\hline Catch tank dimensions & \\
\hline Overall length & \\
\hline & \\
\hline & \\
\hline & \\
\hline
\end{tabular}

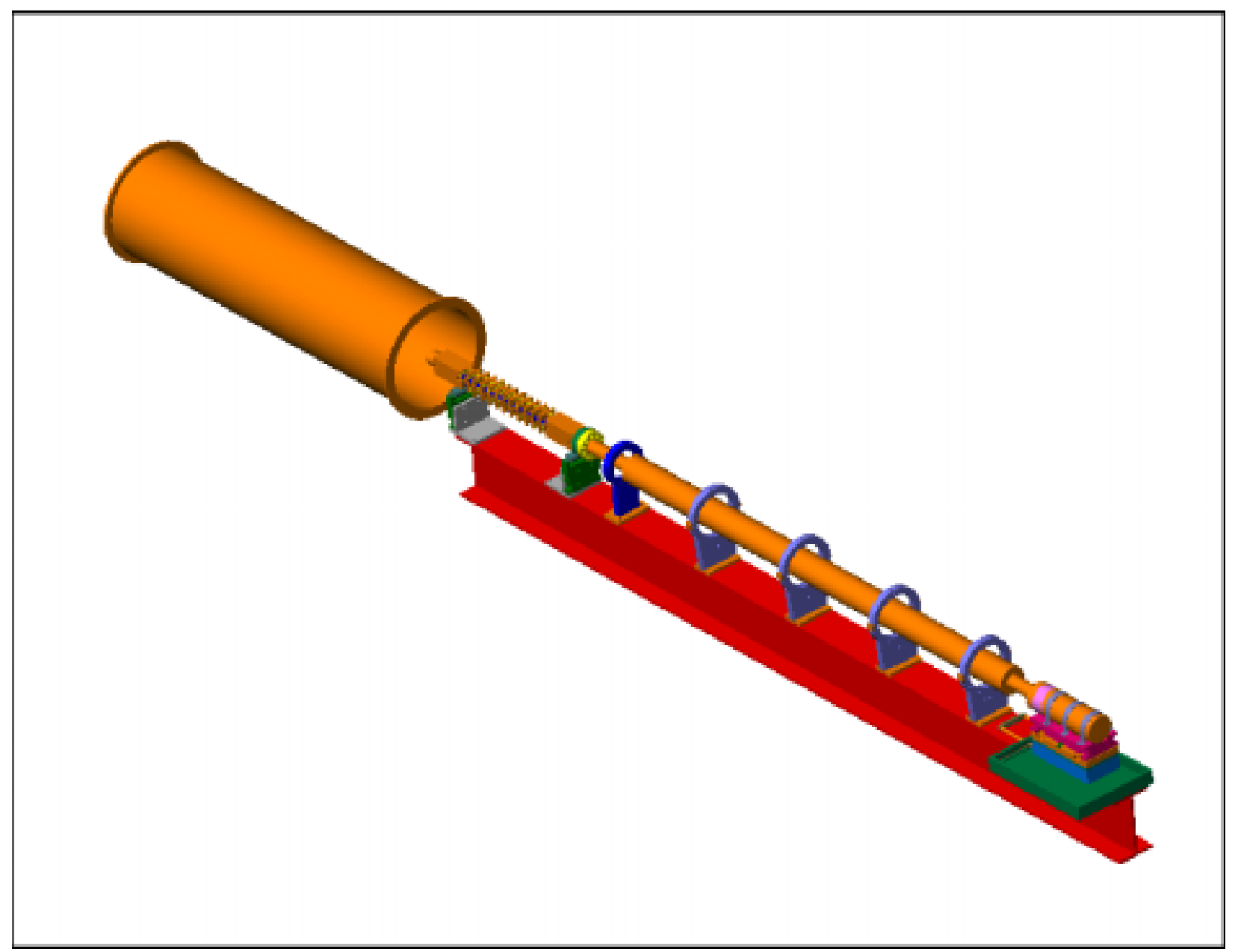

Figure 2. Distributed Injection Launcher System Components. This perspective view shows the Computer Aided Design (CAD) description of some of the assembled hardware. From the right, one sees the helium breech, the helium barrel, the distributed injection section, and the catch tank. (T. M. Uphaus) 


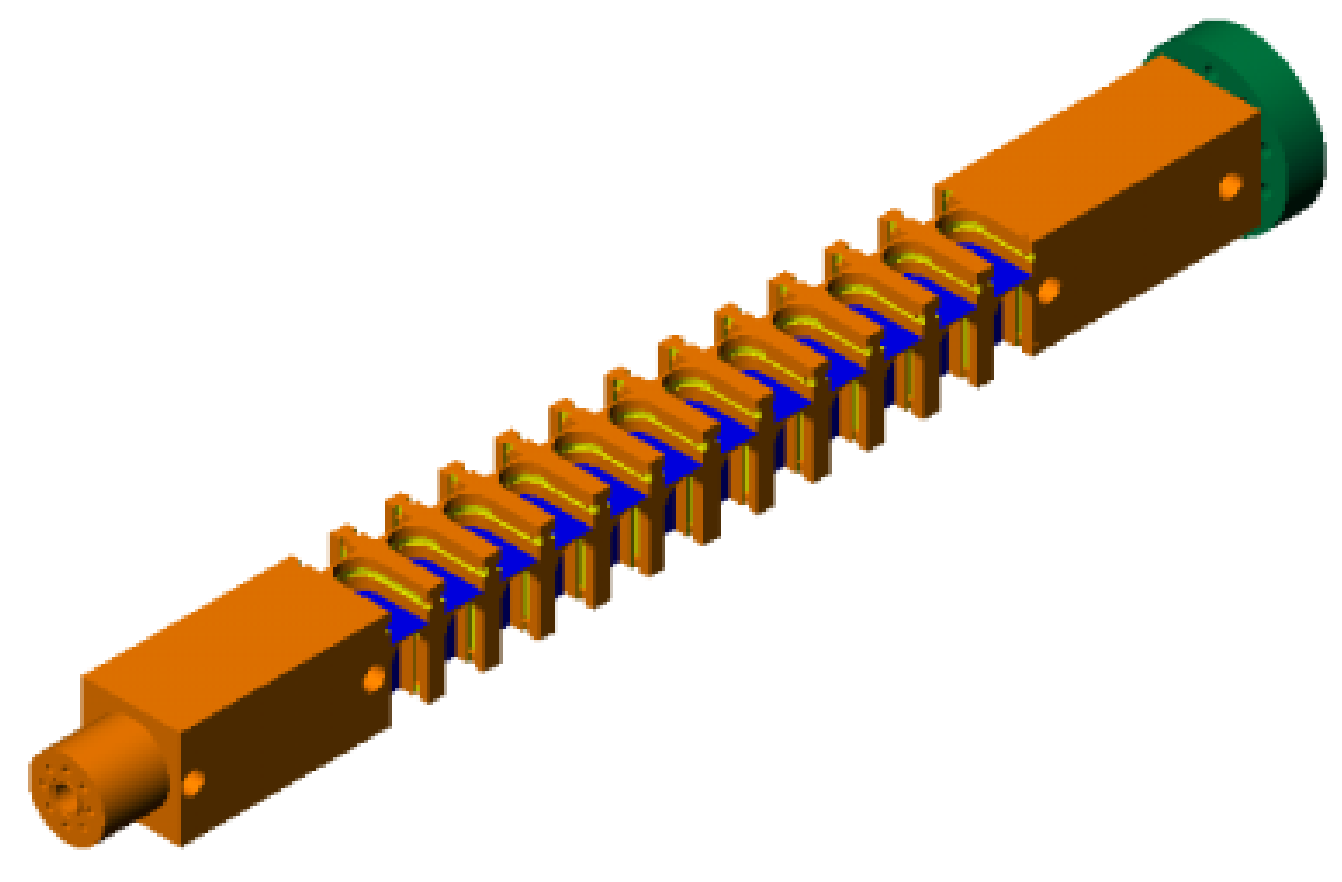

Figure 3. This perspective view shows the CAD description of some of the 12 stage distributed injection section. Each stage uses four symmetrically located replaceable diaphragms. (T. M. Uphaus)

\section{Experimental program}

We designed and built a gas injection test fixture shown schematically below. The fixture comprised three separable internal volumes into which various gasses at various pressures could be introduced. The pressure chamber body volume is separated from the high-pressure test volume by a moveable piston, while the high-pressure test volume was separated from the lowpressure test volume by the replaceable burst diaphragm. We operated the test fixture in a variety of different modes, with different gasses being introduced into different chambers at different time scales using a number of techniques. The purpose of this fixture was twofold. First, we conducted a series of tests to measure the performance of various fast diaphragm-opening methods. Next, we initiated a second test series to measure the time resolved rise in pressure at the far end of the low pressure test volume located $25 \mathrm{~mm}$ from the diaphragm.

In this paper, we focus on a series of tests run with an externally activated diaphragm. In this mode, the piston was withdrawn to the left, the two pressure chambers were supplied with the same high pressure test gas, and the pressure chamber was evacuated to a few hundred $\mathrm{Pa}$ 


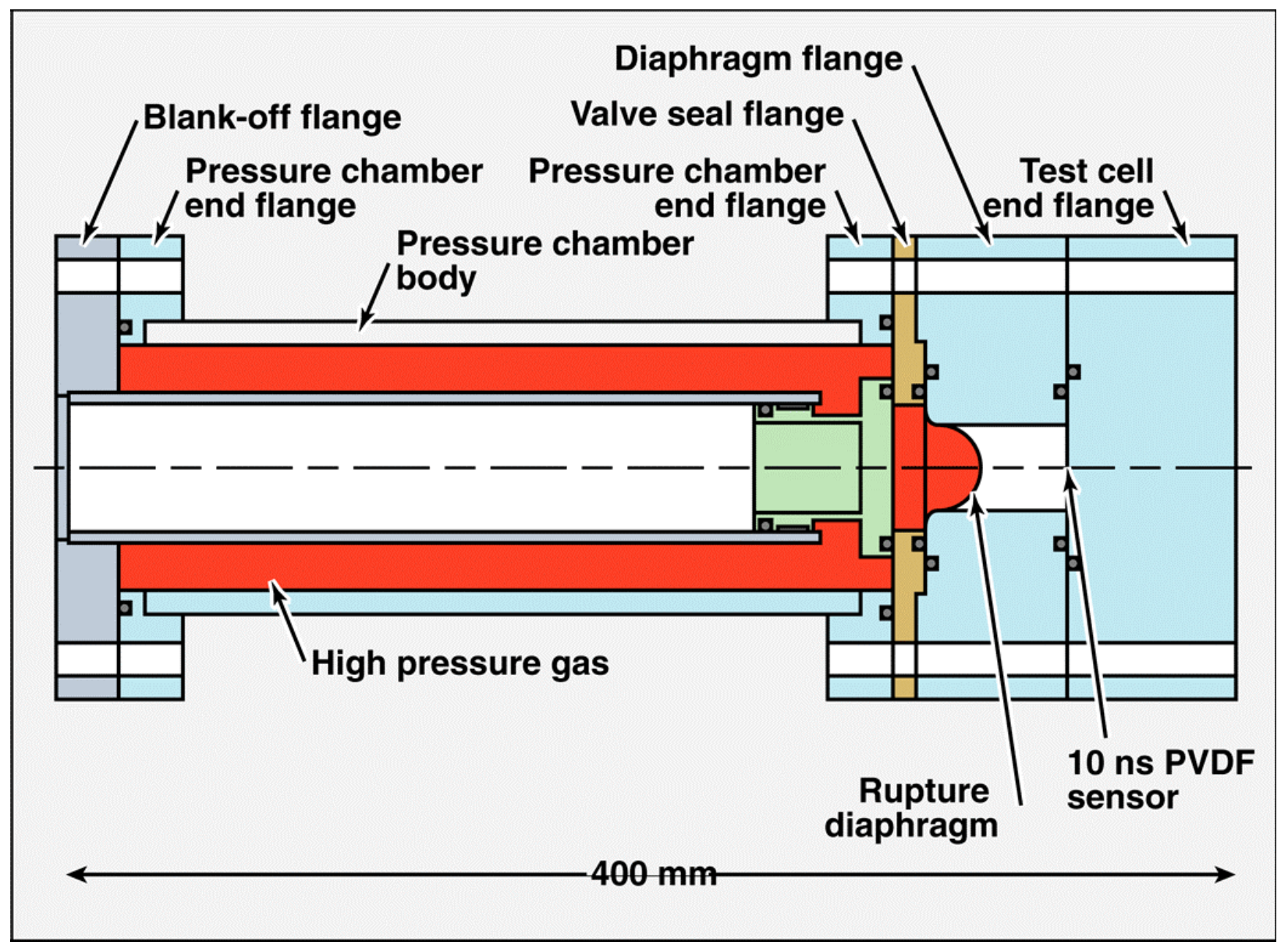

absolute pressure and sealed off. Test conditions are listed in table 2.

Table 2. Summary of typical test parameters

\begin{tabular}{|l|l|}
\hline High pressure: & $0.2 \mathrm{MPa}$ \\
\hline Low pressure: & $100 \mathrm{~Pa}$ \\
\hline Diaphragm material & Kapton \\
\hline Diaphragm thickness & $2 \mathrm{~mm}$ \\
\hline Sensor type & \\
\hline Sensor response whatever & \\
\hline Other stuff & \\
\hline & \\
\hline & \\
\hline
\end{tabular}

\section{Experimental results}

The graph shows the pressure trace taken at the rear wall, $25 \mathrm{~mm}$ from the diaphragm under the conditions described above. The pressure trace begins at time zero, when the signal is sent to fire the diaphragm. After an initial delay, the pressure at the rear wall begins to rise. While the original pressure in the reservoir was $0.2 \mathrm{MPa}$, it can be seen that the pressure measured at the wall, overshoots the reservoir pressure by nearly $50 \%$. The implications of this measurement are potentially dramatic. If one thinks of the rear chamber wall as a simulation of the edge of the sloped boattail on the projectile, we are effectively applying 50\% more pressure to the projectile 
base than is contained in the reservoir. This overshoot phenomenon was repeatedly observed throughout the test program, and was thought to be an artifact of the test setup or instrumentation. Following extensive calibration and bench testing of the gages, we concluded that the effect was real, and ascribable to the direct energy exchange mechanism discovered at the beginning of the twentieth century (5):

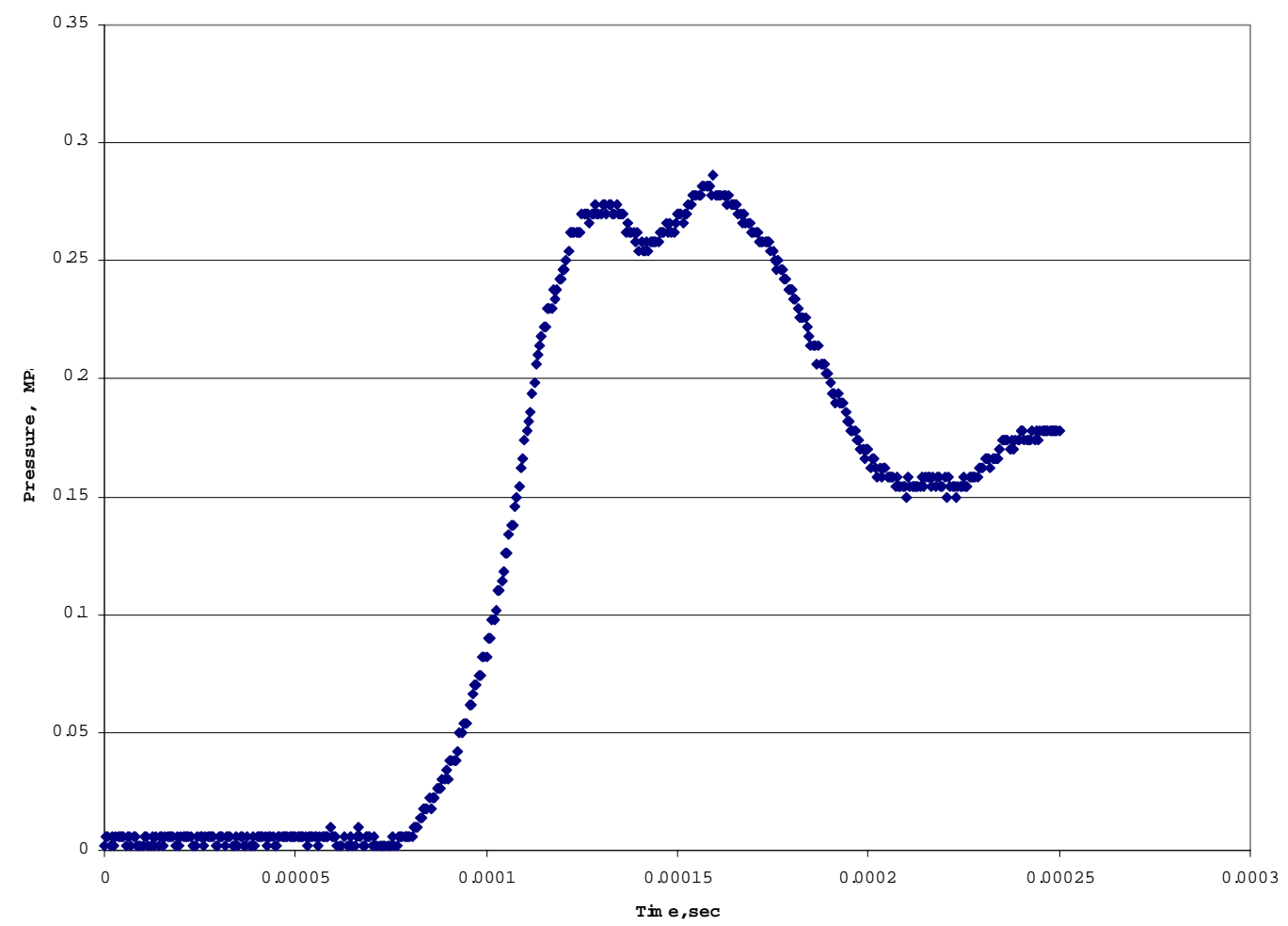

\section{Analytical program}

In concert with the experimental program, we undertook a series of analyses of the test fixture and its geometry. The design concept for the launcher presents formidable challenges to the modeling program. The projectile traverses an evacuated region of a long tube, where the equations of continuum flow are invalid. As the projectile passes, a series of distributed reservoirs of high-pressure gas is released, providing the motive force for acceleration. These conditions produce mixed contiguous volumes of very high and very low pressure gasses surrounded by coupled moving masses. For dilute gas flows, one must use a kinetic theory model such as the Molecular Dynamics approach or the Direct Simulation Monte Carlo (6) method, while the high pressure regime requires the use of the partial differential equations commonly used in hydrodynamics, the Navier-Stokes and Euler equations.

Monte Carlo methods are usually used when the characteristic dimension of the problem is on the order of the mean free path. The MFP for atmospheric conditions is around $50 \mathrm{~nm}$. For the high pressures distributed injection system, the design calls for pressures in the low GPa range, where the mean free path will be around $0.1 \mathrm{~nm}$. For the DSMC method, each cell should be less than 1 mean free path, and each cell should contain at least 50 particles or so (7). Since the physical 
dimensions of the injector would be about $10 \mathrm{~mm}$, the DSMC method becomes impractical, and the continuum approach has to be used. However, at the leading edge of the flow, we are interested in calculating the early time resolved pressure rise against the moving surface of the projectile, at which point the continuum flow model is invalid.

One approach that has been suggested (8) is to ignore the rarefied region and assume that the expansion has a discontinuous leading edge at some low but non zero sound speed. For simple geometries with fixed boundaries, this approach can give a reasonable result for pressure, but fails to properly calculate temperature. One possible solution to this dilemma would be to develop a combined approach that uses a continuum model for the high pressure region and the DSMC method at the high Knudsen number tail of the expansion. Although work is in progress to develop such a method (9), none exists to date. Since the location of the boundary is changing with time, and the geometry is three dimensional, this problem will require large computational resources.

\section{Numerical program}

Because of these known limitations in the analytical capabilities, and to confirm and clarify the experimental results, we employed the ALE hydrocode and constructed a quarter section model of the gas cavities within the test fixture. The first stage calculation shows the pressure rise in a classical shock tube experiment, where the distal end is evacuated and the barrier is suddenly removed. The pressure rises asymtotically to the final value as expected. The pressure does not attain the initial value in the reservoir, because of volume reduction effects.
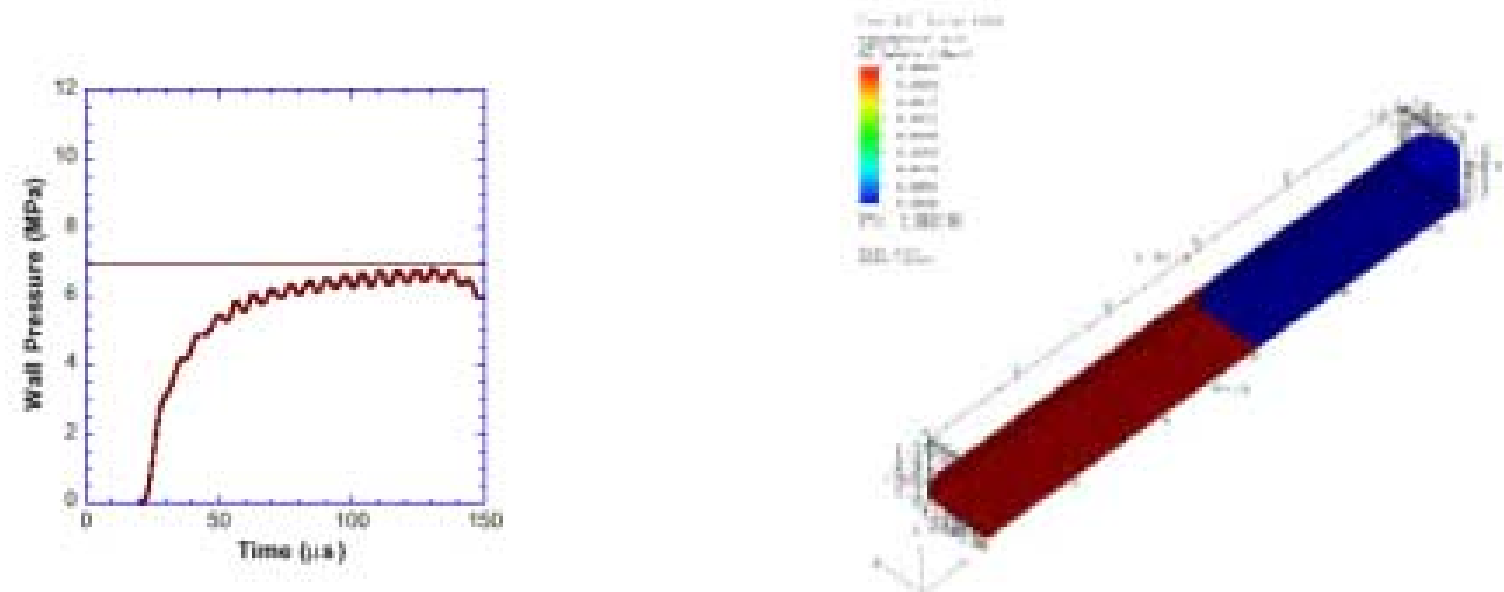

The second series shows the case that the launcher community calls "chambrage"(11). In this result, we can see that the pressure measured at the far end of the small tube is greater than the pressure maintained in the reservoir. In the unsteady fluid mechanics community, this phenomenon is a fundamental process known as "unsteady duct filling" and is used to design pressure amplification systems. Although the advantages of chambrage have been known empirically for centuries, and have been analyzed in various approaches in the launcher community (12), the connection to the unsteady fluid mechanics world has evidently not been made. 

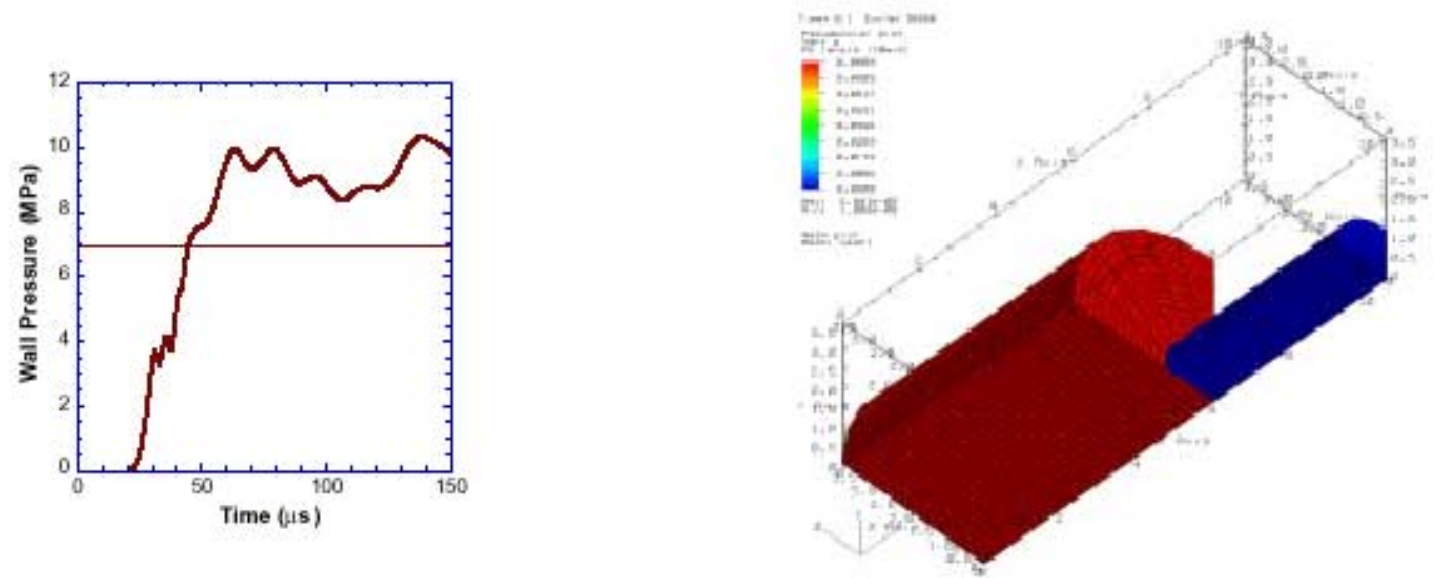

The final picture shows the simulation of the geometry of our test fixture. The stepped geometry of the test cell slows the pressure growth rate slightly and reduces the maximum pressure amplification effect. These results mirror and confirm those of our experimental program and the conclusions of reference 5, p 66.
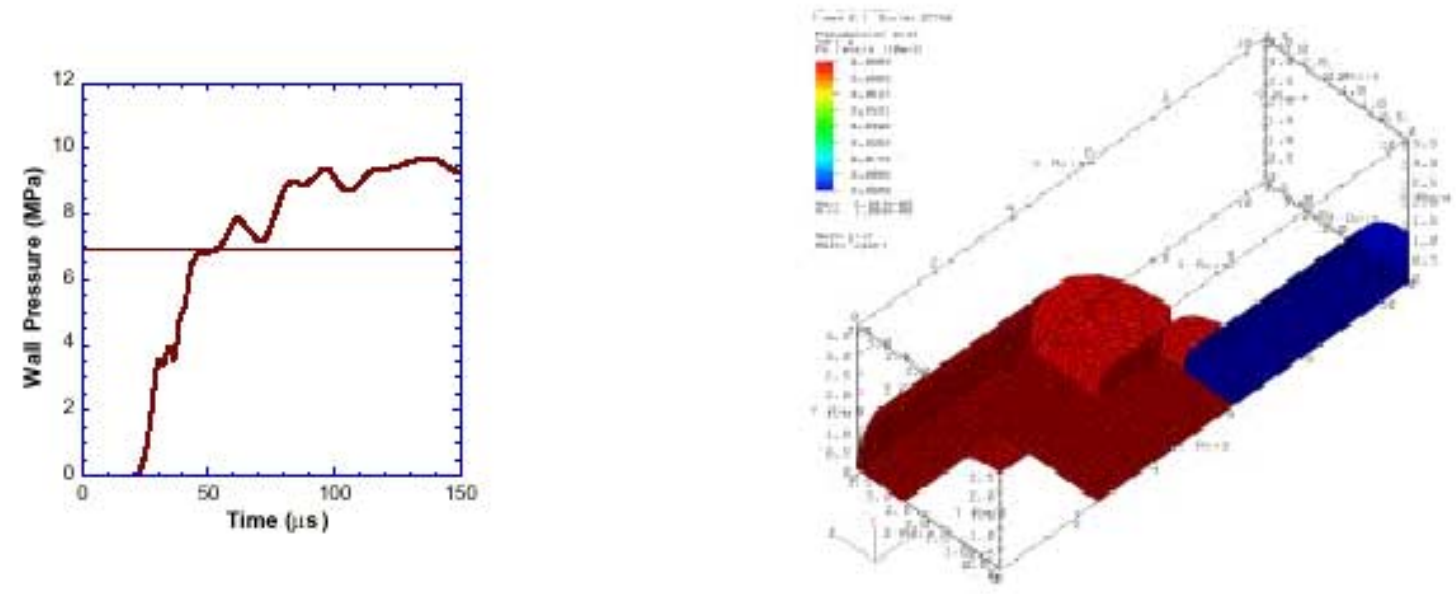

\section{Summary}

Our experimental and analytical program has illuminated a new régime in the field of high velocity gas dynamic launchers. Although many questions and research remains to be carried out, we estimate that a practical, $15 \mathrm{~km} / \mathrm{s}$, high-velocity launcher could be built using our directenergy-exchange, distributed-injection approach. However, the radical nature of the results, the lack of confirming or allied work being carried out anywhere else, and the fact that it would take extensive time and resources to demonstrate targeted performance precluded our further development of this design concept. 


\section{References}

1. Nellis, W. J., 2000, Scientific American

2. Bogdanoff, D.W., "Optimization study of the Ames 0.5" two-stage light gas gun," International Journal of Impact Engineering, 1997, Vol. 20, No. 1-5, pp. 131-142.

3. Higgins, A.J., "A Comparison of Distributed Injection Hypervelocity Accelerators," AIAA-972897, $33^{\text {rd }}$ AIAA/ASME/SAE/ASEE Joint Propulsion Conference and Exhibit, Seattle, WA, 1997

4. Gilreath, H.E., Fristrom, R.M., and Molder, S., "The Distributed -Injection Ballistic Launcher," Johns Hopkins APL Technical Digest, Vol. 9, No. 3, 1998, pp. 299-309.

5. Azoury, P.H. Engineering Applications of Unsteady Fluid Flow, Wiley, 1992.)

6. Bird, G. A., Molecular Gas Dynamics and the Direct Simulation of Gas Flows, Oxford Engineering Science Series, Vol. 42, 1994

7. Alexander, F.J.; and Garcia, A.L, "The direct simulation Monte Carlo method," Computers In Physics, 1997 NOV-DEC, Vol. 11 No. 6, pp. 588-593.

8. Bird, G.A., "Impact of a freely expanding gas on a wall," The Physics of Fluids, Vol. 16, No. 11, pp. 1830-1834, 1973

9. Alder, B., "Highly discretized dynamics,” Physica A, Vol. 240, pp. 193-195, 1997

10. Donea, J., “An Arbitrary Lagrangian-Eulerian Finite Element Method for Transient FluidStructure Interactions,” Comp. Meth.Appl. Mech. Engng., Vol. 33, pp. 689-723, 1982.

11. Glass, I.I., and Sislian, J.P., Nonstationary Flows and Shock Waves, Oxford, 1994.

12. Seigel, A.E., The Theory of High Speed Guns, NATO AGARGograph 91, 1965. 